\title{
Stability, Hydrogen Bond Occupancy Analysis and Binding Free Energy Calculation from Flavonol Docked in DAPK1 Active Site Using Molecular Dynamic Simulation Approaches
}

\author{
Adi Tiara Zikri ${ }^{1,2}$, Harno Dwi Pranowo ${ }^{1,2^{\star}}$, and Winarto Haryadi ${ }^{2}$ \\ ${ }^{1}$ Austrian-Indonesian Centre (AIC) for Computational Chemistry, Faculty of Mathematics and Natural Sciences, \\ Universitas Gadjah Mada, Sekip Utara, Yogyakarta 55281, Indonesia \\ ${ }^{2}$ Department of Chemistry, Faculty of Mathematics and Natural Sciences, Universitas Gadjah Mada, \\ Sekip Utara, Yogyakarta 55281, Indonesia
}

\section{*Corresponding author:}

email:harnodp@ugm.ac.id

Received: May 13, 2020

Accepted: July 22, 2020

DOI: $10.22146 /$ ijc. 56087

\begin{abstract}
Stability and hydrogen bond occupancy analysis of flavonol derivative docked in DAPK1 have been carried out using molecular dynamics simulation approach. Six flavonol derivatives were docked in DAPK1 as protein target, then continued with molecular dynamics simulation. NVT and NPT ensembles were used to equilibrate the system, followed by 20 ns sampling time for each system. Structural stability and hydrogen bond occupancy analyses were carried out at the NVT ensemble, while free binding energy analysis was done at NPT ensemble. From all compounds used in this work, compound $B$ (5,7-dihydroxy-2-(4-hydroxyphenyl)-6-methoxy-4H-chromen-4-one) has a similar interaction with reference ligands (quercetin, kaempferol, and fisetin), and the most stable complex system has the maximum RMSD around $2 \AA$. Compound C complex has $-48.06 \mathrm{~kJ} / \mathrm{mol}$ binding free energy score, and it was slightly different from quercetin, kaempferol, and fisetin complexes. Even though complex $C$ has similar binding free energy with the reference compound, complex $B$ shows more stable interactions due to their hydrogen bond and occupancy.
\end{abstract}

Keywords: flavonol; hydrogen bond occupancy; molecular dynamics simulation

\section{- INTRODUCTION}

Flavonoids are one of the natural polyphenol produced from plant secondary metabolites. In human diet, they are most concentrated in fruits, vegetables, wines, tea, and cocoa. Flavonoids have substantial pharmacological properties such as antioxidant, antiinflammatory, antiproliferative, photoprotective, depigmentation, and anti-aging, which are very useful for skin treatment [1-4]. Flavonoids are benzo- $\gamma$-pyrone derivative consisting of phenolic and pyran rings (Fig. 1).

There is some classification of flavonoids such as flavanols, flavone, flavonol, flavanone, isoflavone, and anthocyanidin. In this work, we use six flavonoids derivative from flavanol class with functional group shown in Table. 1.<smiles>O=c1c(O)c(-c2ccccc2)oc2ccccc12</smiles>

Fig 1. Flavonol analogue

Quercetin (3,3', $4^{\prime}, 5,7$-pentahydroxyflavone), kaempferol (3,4',5,7-tetrahydroxyflavone), and fisetin $\left(3,3^{\prime}, 4^{\prime}, 7\right.$-tetrahydroxyflavone) are flavonol derivative in a number of fruits and vegetables such as strawberry, apple, persimmon, grape, onion, and cucumber. Some studies have shown that fisetin has a beneficial effect against numerous diseases [5-8]. The hydroxy group in fisetin can be used as a radical scavenging agent. Quercetin 
Table 1. Reference compounds and test compounds [4]

\begin{tabular}{|c|c|c|c|}
\hline Compound & IUPAC name & Structure & Similarity score to quercetin \\
\hline Quercetin & $\begin{array}{l}\text { 3,3',4',5,7-pentahydroxy-2-phenylchromen- } \\
\text { 4-one }\end{array}$ & & 1.000 \\
\hline Kaempferol & $\begin{array}{l}\text { 3,4',5,7-tetrahydroxy-2-phenylchromen-4- } \\
\text { one }\end{array}$ & & 0.946 \\
\hline Fisetin & $\begin{array}{l}\text { 3,3',4',7-tetrahydroxy-2-phenylchromen-4- } \\
\text { one }\end{array}$ & & 0.959 \\
\hline A & $\begin{array}{l}\text { 5,7-dihydroxy-2-(2-hydroxyphenyl -6,8- } \\
\text { dimethoxy-4H-chromen-4-one }\end{array}$ & & 0.785 \\
\hline $\mathrm{B}$ & $\begin{array}{l}\text { 5,7-dihydroxy-2-(4-hydroxyphenyl -6- } \\
\text { methoxy-4H-chromen-4-one }\end{array}$ & & 0.804 \\
\hline $\mathrm{C}$ & $\begin{array}{l}\text { 3,5,7-trihydroxy-2-(4-methoxyphenyl -8-(3- } \\
\text { methylbut-2-en-1-yl -4H-chromen-4-one }\end{array}$ & & 0.768 \\
\hline
\end{tabular}

has 5 hydroxy groups, which is more than fisetin and kaempferol. In some works [9-13], it is assumed that the hydroxy group has a positive correlation with radical scavenging agent activity. However, yet no accurate data to prove this hypothesis.

DAPK1 (Death-Associated Protein Kinase) is one of $\mathrm{Ca}^{2+} /$ calmodulin-dependent protein kinase that includes $160 \mathrm{kDA}$ serine/threonine protein kinase [14]. In the earlier study, DAPK1 was identified as a mediator of $\gamma^{-}$ interferon-induced cell death, tumor suppressor, and autophagy activation [15-17]. The structure-binding affinity of DAPK1 and flavonol at the atomic-level could be explained using crystallographic analysis [16]. In the previous work [18], they investigated flavonoids activity in DAPK1 protein [19], but the dynamical properties of flavonol and its stability with water solvent to predict their interaction have not been done yet.

Molecular docking is a computational chemistry method to observe the active site of a protein and its interaction with ligand [20-22]. Their interactions usually involve hydrogen bonds between the ligand and amino acid from protein [23-25]. In this work, flavonol derivatives are ligand, and DAPK1 is the target protein. Subsequently, molecular dynamics simulation is performed to investigate stability, hydrogen bond occupancy, and binding free energy of ligand and protein in an aqueous system.

\section{- COMPUTATIONAL METHODS}

\section{Molecular Docking}

Death-associated protein kinase 1 (DAPK1) was obtained from RCSB protein databank with code: 5AUW. 
The structure was prepared for re-docking using UCSF Chimera 1.12 [26]. Quercetin in DAPK1 was redocked using PLANTS 1.2 [27], followed by fisetin and kaempferol docking. Compound $\mathrm{A}, \mathrm{B}$, and $\mathrm{C}$ were obtained from drugbank.ca with a similarity structure score above 0.7 from quercetin structure [28]. Ten conformations were formed by this docking method. The conformation that has similar conformation with quercetin was used to analyze their interaction. The interaction of flavonoid compound and amino acid were shown in Table 2. The length of interaction was in the range of 1.6-2.4 $\AA$. This distance indicated a hydrogen bond interaction was formed. Hydrogen bond analysis was generated using VMD [29].

\section{Simulation Protocol}

Structure and dynamics of flavonoid, protein, and water were studied using molecular dynamics (MD) simulation. A cubic simulation box [30] was built with a volume of $589.89 \mathrm{~nm}^{3}$. In this box, 1 flavonoid compound and 1 DAPK1 were immersed in 18,183 water molecules by employing SPC water model. GROMOS 54A7 force field [31-32] was used in this simulation method. Steepest descent algorithm was performed to obtain minimization energy followed by equilibration in NVT and NPT ensemble for 250 ps (125,000 steps) and 500 ps $(250,000$ steps) with a time step of 2 fs. A periodic boundary condition to all three-dimensional space was applied. To integrate the equation of motion, a leapfrog integrator was employed. The temperature was kept at $300.15 \mathrm{~K}$ using a V-rescale Berendsen thermostat [33]. The pressure was kept constant at 1 bar by employing Parrinello-Rahman pressure coupling. Particle Mesh Ewald (PME) [34], and it was used to correct the long electrostatic interaction as well. MD production data of simulation was generated in 20 ns (10,000,000 steps) for each simulation system. Binding Free energy was calculated using the Free Energy Perturbation (FEP) method for solvated complex [35-36].

\section{- RESULTS AND DISCUSSION}

\section{Molecular Docking Interaction}

In this work, DAPK1 was downloaded from the protein data bank. Quercetin was already available inside the DAPK1 active site. Redocking was performed to determine the ligand coordinate inside DAPK1. This coordinate was then used to perform molecular docking of reference and test compounds.

Fig. 2 shows the overlapping of all flavonol compounds in the DAPK1 active site. Quercetin and

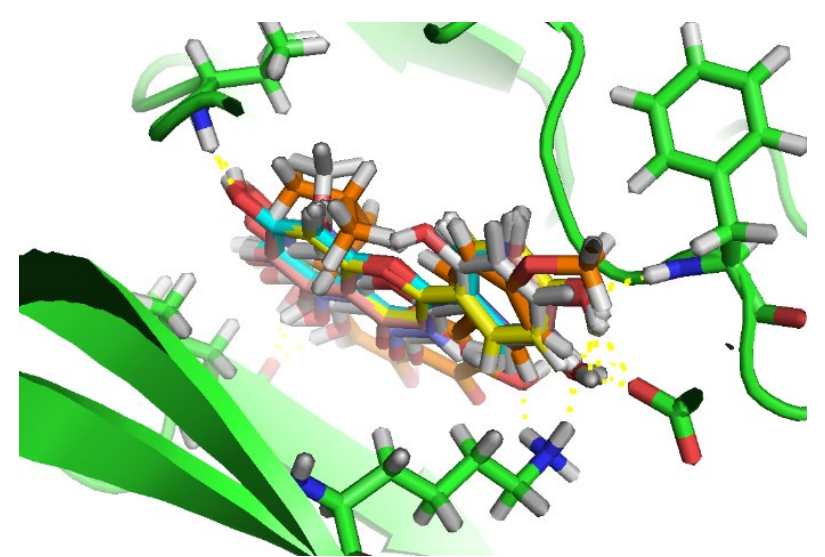

Fig 2. Visualization of flavonol inside DAPK 1 active site

Table 2. Residue interaction between flavonol and DAPK1

\begin{tabular}{lcc}
\hline Compound & Residue interaction & Distance $(\AA)$ \\
\hline Quercetin & VAL 95 & 2.1 \\
& VAL 95 & 1.6 \\
& PHE 159 & 2.1 \\
& GLU 63 & 1.8 \\
& GLU 63 & 1.6 \\
Kaempferol & LYS 41 & 2.0 \\
& VAL 95 & 2.1 \\
& VAL 95 & 1.7 \\
& PHE 159 & 2.1 \\
Fisetin & GLU 63 & 1.9 \\
& VAL 95 & 2.1 \\
& VAL 95 & 1.7 \\
& PHE 159 & 2.2 \\
& GLU 63 & 1.7 \\
A & GLU 63 & 1.8 \\
& LYS 41 & 1.9 \\
B & VAL 95 & 2.1 \\
& LEU 18 & 2.0 \\
& VAL 95 & 1.7 \\
& VAL 95 & 2.1 \\
& PHE 159 & 2.2 \\
C & GLU 63 & 1.7 \\
& LEU 18 & 2.3 \\
& ASP 158 & 2.4 \\
& LYS 41 & 2.0 \\
& LEU 18 & 1.9 \\
\hline
\end{tabular}


fisetin have 6 interactions with distance 1.6 to $2.4 \AA$ (see Table 2).

The amino acids that interact with quercetin and fisetin are LYS 41, GLU 63, PHE 159, and VAL 95, where GLU63 takes a role as the amino acid with the closest interaction with quercetin and fisetin. Meanwhile, there are 4 interactions in kaempferol, which the interaction to LYS 41 and one interaction with GLU 63 is unidentified. Compound $\mathrm{A}, \mathrm{B}$, and $\mathrm{C}$ give significant difference interaction in molecular docking. New interactions are observed after docking process. LEU 18 forms an interaction to compound $\mathrm{A}, \mathrm{B}$, and $\mathrm{C}$. Interaction with ASP 158 is only formed in compound C. Most of the interactions are unidentified because of the position of the hydroxy functional group. The more occurred interactions between flavonol will enhance the strength of flavonol binding. From the molecular docking data, compound B has nearly similar interaction to the DAPK1 active site with quercetin, kaempferol, and fisetin. The similar interaction between flavonol and DAPK1 indicates that the conformation structure of flavonol is not much different.

\section{Molecular Dynamics Simulation}

The stability of flavonol complexes with DAPK1 shown in Fig. 3. RMSDs of each system are combined to see the difference from that.

Kaempferol complex has the highest RMSD of all systems when the simulation time exceeds 15 ns. After $15 \mathrm{~ns}$, RMSD values reach $3.8 \AA$, and this RMSD score increased as the simulation run got longer. It indicates that the kaempferol complex has less stability during the simulation. For the fisetin complex, the RMSD value is relatively lower than other complexes, which assumed that the complex might be stable. However, some atoms in the fisetin complex that have high RMSF values make RMSD distance be fluctuating. The fisetin complex becomes less stable at simulation time 12 and 18 ns with RMSD value of $2.5 \AA$. The quercetin complex has constant RMSD with an average value of $3 \AA$. The highest RMSD value of $3.5 \AA$ was found at 6 and 16 ns. From the new compound complexes, compound $\mathrm{B}$ complex has the lowest RMSD with an average value of $2.3 \AA$. The RMSD of compound B complex reaches its maximum value of $3.2 \AA$ at $7,12,15$, and $17 \mathrm{~ns}$. Although the backbone distance of the compound $\mathrm{B}$ complex is getting higher, it immediately becomes lower at once. It indicates that compound $\mathrm{B}$ complex forms a stable complex with protein. This trend takes effect in hydrogen occupancy, where the more occupancy of complex, the more stable the complex is. For other complex, compound $\mathrm{A}$ is the least stable compare to those three compounds with an average RMSD value of $2.7 \AA$, followed by compound C complex with an average RMSD value of $2.5 \AA$.

\section{Hydrogen bond occupancy of quercetin, fisetin, and kaempferol during simulation}

Hydrogen bond occupancy is analyzed using VMD package and is depicted in Fig 4. Fisetin has 15 hydrogen
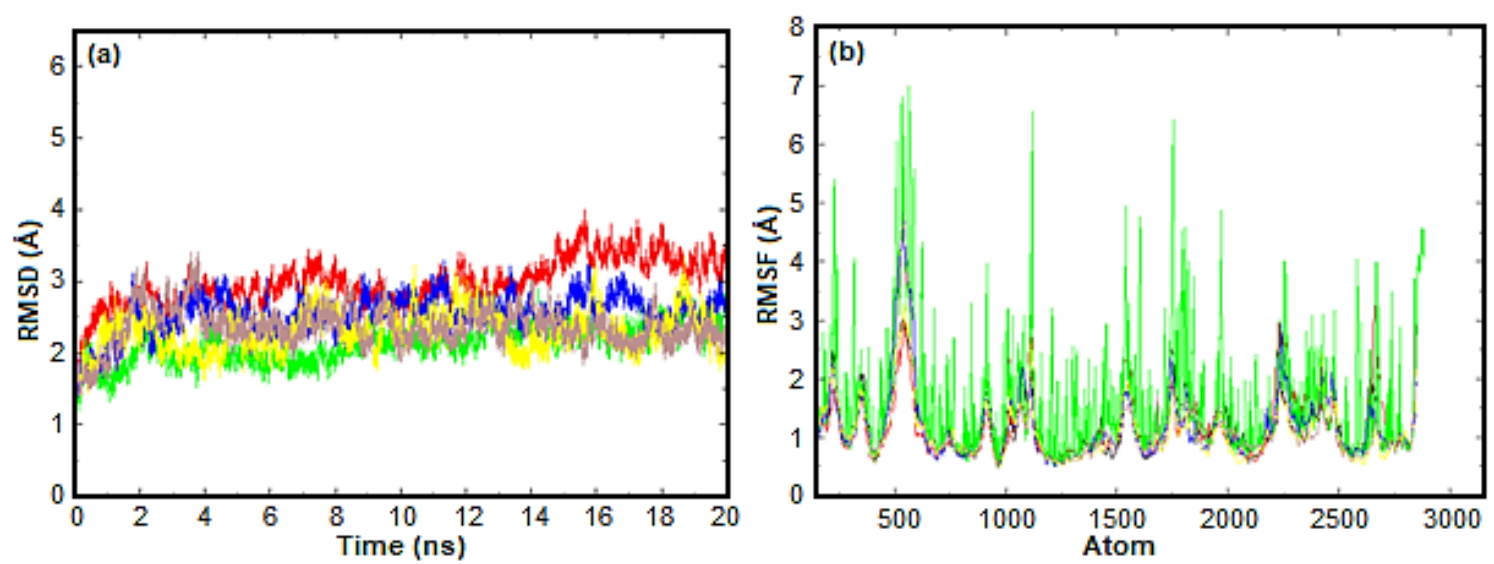

Fig 3. (a) RMSD and (b) RMSF of quercetin (black), kaempferol (red), fisetin (green), compound A (blue), compound B (yellow), compound C (brown) with DAPK1 


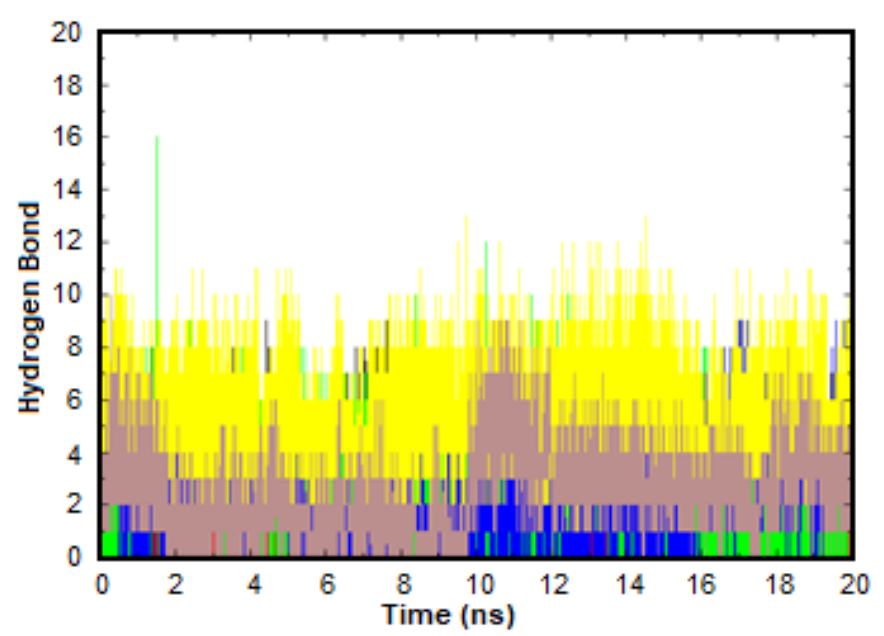

Fig 4. Hydrogen bond of quercetin (black), kaempferol (red), fisetin (green), compound A (blue), compound B (yellow), compound C (brown) with DAPK1 amino acid

bonds in a very short time at the beginning of simulation time, and compound $\mathrm{B}$ has the largest total hydrogen bond among test compounds. Compound $\mathrm{A}$ and compound $\mathrm{C}$ only have 8 hydrogen bonds. Table 3 lists the three most stable hydrogen bonds of each complex.

For reference compound, quercetin has 57 total hydrogen bonds with the highest occupancy of $26.04 \%$, where quercetin forms the hydrogen bond with GLY 98.
Then $15.47 \%$ occupancy where quercetin plays a role as a donor to LEU 92 and $14.62 \%$ with LEU 144 . Kaempferol has 39 total hydrogen bonds with the highest occupancy of $29.91 \%$, where kaempferol and GLY 97 form hydrogen bonds. Then 19.15\% occupancy where kaempferol plays a role as a donor to GLU 99 and $15.49 \%$ with ILE 76 . Fisetin has 48 total hydrogen bonds with the highest occupancy of $24.61 \%$, where fisetin has the role of a donor and GLY as an acceptor of hydrogen bond formation 19 . Then $21.07 \%$ occupancy where fisetin plays a role as a donor to ASP 158 and $20.90 \%$ with GLU 140. For the test compound, compound A has 39 total hydrogen bonds with the highest occupancy of $49.69 \%$, where compound A and GLU 99 have a high probability of forming hydrogen bonds. Then $29.71 \%$ occupancy where compound A plays a role as a donor to MET 143 and $26.87 \%$ where compound A form hydrogen bonds with ASP 158. Compound B has 30 total hydrogen bonds with the highest occupancy of $212.74 \%$, where compound B has a high probability with GLU 63 to form hydrogen bonds. Then $132.71 \%$ occupancy where compound B plays a role as a donor to GLU 93, and 59.66\% with compound B interact with PHE 159 and form hydrogen bonds. Compound $\mathrm{C}$ has 37 total hydrogen bond with the

Table 3. Hydrogen bond occupancy

\begin{tabular}{lllc}
\hline Compound & \multicolumn{1}{c}{ Donor } & \multicolumn{1}{c}{ Acceptor } & Occupancy $\%$ \\
\hline Quercetin & QUE 275-Side & GLY 98-Main & 26.60 \\
& QUE 275-Side & LEU 92-Side & 15.47 \\
\multirow{3}{*}{ Kaempferol } & QUE 275-Side & LEU 144-Main & 14.62 \\
& GLY 97-Main & KAEM 275-Side & 29.91 \\
& KAEM 275-Side & GLU 99-Side & 19.15 \\
\multirow{2}{*}{ Fisetin } & KAEM 275-Side & ILE 76-Side & 15.49 \\
& FISE 275-Side & GLY 19-Main & 24.61 \\
& FISE 275-Side & ASP 158-Side & 21.07 \\
A & FISE 275-Side & GLU 140-Side & 20.90 \\
& Com A 275-Side & GLU 99-Side & 49.69 \\
& Com A 275-Side & MET 143-Side & 29.71 \\
B & ASP 158-Main & Com A 275-Side & 26.87 \\
& Com B 275-Side & GLU 63-Side & 212.74 \\
& Com B 275-Side & GLU 93-Main & 132.71 \\
C & PHE 159-Main & Com B 275-Side & 59.66 \\
& Com C 275-Side & GLU 99-Side & 135.91 \\
& ASP 158-Main & Com C 275-Side & 33.71 \\
& GLU 99-Main & Com C 275-Side & 19.57 \\
\hline
\end{tabular}


Table 4. Free energies of hydrated ligands, protein - ligand complexes and binding energies of flavonol complexes

\begin{tabular}{lccc}
\hline \multicolumn{1}{c}{ Compound } & $\Delta \mathrm{G}$ Complex $(\mathrm{kJ} / \mathrm{mol}$ & $\Delta \mathrm{G}$ Ligand $(\mathrm{kJ} / \mathrm{mol}$ & $\Delta \mathrm{G}$ Binding $(\mathrm{kJ} / \mathrm{mol}$ \\
\hline Quercetin & -85.69 & -46.39 & -39.3 \\
Kaempferol & -90.98 & -45.06 & -45.92 \\
Fisetin & -110.36 & -63.7 & -46.66 \\
Compound A & -87.01 & -78.87 & -8.14 \\
Compound B & -78.48 & -49.51 & -28.97 \\
Compound C & -92.39 & -44.33 & -48.06 \\
\hline
\end{tabular}

highest occupancy $135.91 \%$ where compound A form hydrogen bond with GLU 99. Then $33.71 \%$ occupancy where compound C play a role as acceptor from ASP 158 and $19.57 \%$ where compound $C$ form hydrogen bond with GLU 99. Hydrogen bond occupancy with a score of more than $100 \%$ indicates that more than one atom pair interacts to form hydrogen bonds. For reference compounds, fisetin has more hydrogen bond and hydrogen bond occupancy than test compounds. Meanwhile, compound B has less total hydrogen bond but has the highest hydrogen bond occupancy. This total hydrogen bond and hydrogen bond occupancy determine the stability of each system. The number and occupancy of hydrogen bonds are the keys to the interaction stabilization of protein - ligand complex.

\section{Free binding energy}

In the molecular dynamic simulation, free energy calculation takes a major role in determining the binding energy of ligand inside protein [24]. In this work, the free energy perturbation (FEP) method was used to calculate binding energy. In this approach, the dummy ligand is involved in changing partial charge in ligand into Lennard-Jones potential [30]. Lambda factor is used for smoothing the transition from dummy to the real ligand in the protein - ligand complex. In this work, the lambda value is ranging from 0 to 10 . Binding Free energies score are presented in Table 4.

Compound $\mathrm{C}$ complex has the most negative binding free energy of $-48.06 \mathrm{~kJ} / \mathrm{mol}$. This energy has slightly different from the reference compound, and it means that the interaction in compound $\mathrm{C}$ complex is the most stable interaction during simulation followed by protein - compound B complex. Although compound B complex has more hydrogen occupancy than compound
C complex as explained above, the compound C complex has more stable interaction than compound $\mathrm{B}$. Compound A complex has the most positive binding energy, indicating that the interaction between compound C in DAPK1 active site is the weakest.

\section{- CONCLUSION}

In this work, stability, hydrogen occupancy, and binding free energy of several protein - flavonol complexes had been investigated through molecular dynamics simulation. For 20 ns of production time, complex compound $\mathrm{B}$ has the most stable structure during simulation with RMSD value of $2.5 \AA$ and the highest hydrogen bonds with $212.74 \%$ occupancy against GLU 63. Even though the binding energy of compound $\mathrm{B}$ is higher than compound $\mathrm{C}$, compound $\mathrm{B}$ has an upper head against other test compounds in terms of stability and hydrogen bond occupancy.

\section{- REFERENCES}

[1] Heim, K.E., Tagliaferro, A.R., and Bobilya, D.J., 2002, Flavonoid antioxidant: Chemistry, metabolism and structure activity relationship, J. Nutr. Biochem., 13 (10), 572-584.

[2] Bucar, F., Wube, A., and Schmid, M., 2013, Natural product isolation - How to get from biological material to pure compounds, Nat. Prod. Rep., 30 (4), 525-545.

[3] Rha, C.S., Jeoung, H.W., Park, S., Lee, S., Jung, Y.S., and Kim, D.O., 2019, Antioxidative, antiinflammatory, and anticancer effects of purified flavonol glycosides and aglycones in green tea, Antioxidants, 8 (8), 278.

[4] Yokoyama, T., Kosaka, Y., and Mizuguchi, M., 2015, Structural insight into the interactions 
between death-associated protein kinase 1 and natural flavonoids, J. Med. Chem., 58 (18), 7400-7408.

[5] Chen, L.Z., Yao, L., Jiao, M.M., Shi, J.B., Tan, Y., Ruan, B.F., Hua, X., and Liu, 2019, Novel resveratrolbased flavonol derivatives: Synthesis and antiinflammatory activity in vitro and in vivo, Eur. J. Med. Chem., 175, 114-128.

[6] Bai, J., Zhao, S., Fan, X., Chen, Y., Zou, X., Hu, M., Wang, B., Jin, J., Wang, X., Hu, J., Zhang, D., and Li, Y., 2019, Inhibitory effects of flavonoids on Pglycoprotein in vitro and in vivo: Food/herb-drug interactions and structure-activity relationships, Toxicol. Appl. Pharmacol., 369, 49-59.

[7] Serafini, M., Peluso, I., and Raguzzini, A., 2010, Flavonoids as anti-inflammatory agents, Proc. Nutr. Soc., 69 (3), 273-278.

[8] Bakoyiannis, I., Daskaloupoulou, A., Pergialiotis, V., and Perrea, D., 2019, Phytochemicals and cognitive health: Are flavonoids doing the trick?, Biomed. Pharmacother., 109, 1488-1497.

[9] Hatahet, T., Morille, M., Hommos, A., Devoisselle, J.M., Müller, R.H., and Bégu, S., 2016, Quercetin topical application, from conventional dosage forms to nanodosage forms, Eur. J. Pharm. Biopharm., 108, 41-53.

[10] Hatahet, T., Morille, M., Hommoss, A., Dorandeu, C., Müller, R.H., and Bégu, S., 2016, Dermal quercetin smartCrystals ${ }^{\circledR}$ : Formulation development, antioxidant activity and cellular safety, Eur. J. Pharm. Biopharm., 102, 51-63.

[11] Rho, H.S., Ghimeray, A.K., Yoo, D.S., Ahn, S.M., Kwon, S.S., Lee, K.H., Cho, D.H., and Cho, J.Y., 2011, Kaempferol and kaempferol rhamnosides with depigmenting and anti-inflammatory properties, Molecules, 16 (4), 3338-3344.

[12] Nagula, R.L., and Wairkar, S., 2019, Recent advances in topical delivery of flavonoids: A review, $J$. Controlled Release, 296, 190-201.

[13] Fan, M., Ding, H., Zhang, G., Hu, X., and Gong, D., 2019, Relationships of dietary flavonoid structure with its tyrosinase inhibitory activity and affinity, LWT Food Sci. Technol., 107, 25-34.
[14] Tu, W., Xu, X., Pheng, L., Zhong, S., Zhang, W., Soudarapandian, M.M., Belal, C., Wang, M., Jia, N., Zhang, W., Lew, F., Chan, S.L., Chen, Y., and Lu, Y., 2010, DAPK1 interaction with NMDA receptor NR2B subunits mediates brain damage in stroke, Cell, 140 (2), 222-234.

[15] Chen, Z., Picaud, S., Filippakopoulos, P., D'Angiolella, V., and Bullock, A.N., 2019, Structural basis for recruitment of DAPK1 to the KLHL20 E3 ligase, Structure, 27 (9), 1395-1404.e4.

[16] Xu, L.Z., Li, B.Q., and Jia, J.P., 2019, DAPK1: A novel pathology and treatment target for Alzheimer's disease, Mol. Neurobiol., 56 (4), 2838-2844.

[17] Park, G.B., Jeong, J.Y., and Kim, D., 2019, Gliotoxin enhances autophagic cell death via the DAPK1TAp63 signaling pathway in paclitaxel-resistant ovarian cancer cells, Mar. Drugs, 17 (7), 412.

[18] Wei, R., Zhang, L., Hu, W., Wu, J., and Zhang, W., 2019, Long non-coding RNA AK038897 aggravates cerebral ischemia/reperfusion injury via acting as a ceRNA for miR-26a-5p to target DAPK1, Exp. Neurol., 314, 100-110.

[19] Singh, P., and Talwar, P., 2017, Exploring putative inhibitors of Death Associated Protein Kinase 1 (DAPK1) via targeting Gly-Glu-Leu (GEL) and Pro-Glu-Asn (PEN) substrate recognition motifs, $J$. Mol. Graphics Modell., 77, 153-167.

[20] Shoichet, B.K., Kuntz, I.D., and Bodian, D.L., 1992, Molecular docking using shape descriptor, J. Comput. Chem., 13 (3), 380-397.

[21] Pinzi, L., and Rastelli, G., 2019, Molecular docking: Shifting paradigms in drug discovery, Int. J. Mol. Sci., 20 (18), 4331.

[22] Torres, P.H.M., Sodero, A.C.R., Jofily, P., and Silva Jr., F.P., 2019, Key topics in molecular docking for drug design, Int. J. Mol. Sci., 20 (18), 4574.

[23] Li, J., Fu, A., and Zhang, L., 2019, An overview of scoring functions used for protein-ligand interactions in molecular docking, Interdiscip. Sci., 11 (2), 320-328.

[24] Turkan, F., Cetin, A., Taslimi, P., Karaman, M., and Gulçin, I., 2019, Synthesis, biological evaluation and 
molecular docking of novel pyrazole derivatives as potent carbonic anhydrase and acetylcholinesterase inhibitors, Bioorg. Chem., 86, 420-427.

[25] Saikia, S., and Bordoloi, M., 2019, Molecular docking: Challenges, advances and its use in drug discovery perspective, Curr. Drug Targets, 20 (5), 501-521.

[26] Pettersen, E.F., Goddard, T.D., Huang, C.C., Couch, G.S., Greenblatt, D.M., Meng, E.C., and Ferrin, T.E., 2004, UCSF Chimera-A visualization system for exploratory research and analysis, J. Comput. Chem., 25 (13), 1605-1612.

[27] Korb, O., Stützle, T., and Exner, T.E., 2006, PLANTS: Application of ant colony optimization to structurebased drug design, Lect. Notes Comput. Sci., 4150, 247-258.

[28] Hodgkin, E.E., and Richards, W.G., 1987, Molecular similarity based on electrostatic potential and electric field, Int. J. Quantum Chem., 32 (S14), 105-110.

[29] Humphrey, W., Dalke, A., and Schulten, K., 1996, VMD-Visual molecular dynamics, J. Mol. Graphics, 14 (1), 33-38.

[30] de Leeuw, S.W., Perram, J.W., and Smith, E.R., 1980, Simulation of electrostatic systems in periodic boundary conditions. I. Lattice sums and dielectric constants, Proc. R. Soc. London, Ser. A, 373 (1752), 27-56.

[31] Silva, T.F.D., Vila-Viçosa, D., Reis, P.B.P.S., Victor, B.L., Diem, M., Oostenbrink, C., and Machuqueiro, M., 2018, The impact of using single atomistic longrange cutoff schemes with the GROMOS 54A7 force field, J. Chem. Theory Comput., 14 (11), 5823-5833.

[32] Berendsen, H.J.C., van der Spoel, D., and van Drunen, R., 1995, GROMACS: A message-passing parallel molecular dynamics implementation, Comput. Phys. Commun., 91 (1-3), 43-56.

[33] van Gunsteren, W.F., and Berendsen, H.J.C., 1977, Algorithms for macromolecular dynamics and constraint dynamics, Mol. Phys., 34 (5), 1311-1327.

[34] Darden, T., York, D., and Pedersen, L., 1993, Particle mesh Ewald: An $N \cdot \log (N)$ method for Ewald sums in large systems, J. Chem. Phys., 98 (12), 10089.

[35] Bash, P.A., Singh, U.C., Langridge, R., and Kollman, P.A., 1987, Free energy calculation by computer simulation, Science, 236 (4801), 564-568.

[36] Brandsal, B.O., Österberg, F., Almlöf, M., Feierberg, I., Luzhkov, V.B., and Åqvist, J., 2003, Free energy calculations and ligand binding, Adv. Protein Chem., 66, 123-158. 